\title{
Obatoclax Regulates the Proliferation and Fusion of Osteoclast Precursors through the Inhibition of ERK Activation by RANKL
}

\author{
Ju Hee Oh ${ }^{1,2}$, Jae Yoon Lee ${ }^{1,2}$, Jin Hyeong Park', Jeong Hyeon No', and Na Kyung Lee,
}

\begin{abstract}
Obatoclax, a pan-Bcl2 inhibitor, shows antitumor activities in various solid malignancies. Bcl2-deficient mice have shown the importance of $\mathrm{Bcl} 2$ in osteoclasts, as the bone mass of the mice was increased by the induced apoptosis of osteoclasts. Despite the importance of Bcl2, the effects of obatoclax on the proliferation and differentiation of osteoclast precursors have not been studied extensively. Here, we describe the anti-proliferative effects of obatoclax on osteoclast precursors and its negative role on fusion of the cells. Stimulation with low doses of obatoclax significantly suppressed the proliferation of osteoclast precursors in a dose-dependent manner while the apoptosis was markedly increased. Its stimulation was sufficient to block the activation of ERK MAP kinase by RANKL. The same was true when PD98059, an ERK inhibitor, was administered to osteoclast precursors. The activation of JNK1/2 and p38 MAP kinase, necessary for osteoclast differentiation, by RANKL was not affected by obatoclax. Interesting$l y$, whereas the number of TRAP-positive mononuclear cells was increased by both obatoclax and PD98059, fused, multinucleated cells larger than $100 \mu \mathrm{m}$ in diameter containing more than 20 nuclei were completely reduced. Consistently, obatoclax failed to regulate the expression of osteoclast marker genes, including c-Fos, TRAP, RANK and CtsK. Instead, the expression of DC-STAMP and Atp6v0d2, genes that regulate osteoclast fusion, by RANKL was significantly abrogated by both obatoclax and PD98059. Taken together, these results suggest that obatoclax down-regulates the proliferation and fusion of osteoclast precursors through the inhibition of the ERK1/2 MAP kinase pathway.
\end{abstract}

${ }^{1}$ Department of Biomedical Laboratory Science, College of Medica Sciences, Soonchunhyang University, Chungnam, 336-745, Korea, ${ }^{2}$ These authors contributed equally to this work.

${ }^{*}$ Correspondence: nlee@sch.ac.kr

Received 11 December, 2014; revised 1 January, 2015; accepted 5 January, 2015; published online 4 February, 2015

Keywords: ERK1/2 MAP kinase, fusion, obatoclax, osteoclast precursors, proliferation

\section{INTRODUCTION}

Bone undergoes a remodeling process that is efficiently controlled by the functional balance of bone formation by osteoblasts and destruction of mineralized bone matrix by osteoclasts (Harada and Rodan, 2003; Teitelbaum, 2000). While osteoblasts are derived from a mesenchymal lineage, osteoclasts are from hemopoietic progenitors of the monocyte/macrophage lineage (Harada and Rodan, 2003; Teitelbaum, 2000). The binding of receptor activator of nuclear factor- $\mathrm{KB}(\mathrm{NF}-\mathrm{\kappa B})$ ligand (RANKL) to its receptor, RANK, on osteoclast precursors is critical for osteoclast differentiation (Anderson et al., 1997; Wong et al., 1997). The elevation in numbers and/or activity of osteoclasts causes bone-related diseases such as Paget's disease, osteoporosis and lytic bone metastasis (Boyle et al., 2003; Rodan and Martin, 2000).

The activation of RANK by RANKL leads to distinct signaling cascades mediated by c-Jun N-terminal Kinase 1/2 (JNK1/2), p38 and extracellular signal-regulated kinase 1/2 (ERK1/2) MAP kinases regulated by the activation of MEK1 in osteoclast precursors, which are critical for RANKL-induced osteoclast differentiation (Boyle et al., 2003; David et al., 2002; Li et al., 2002; Wei et al., 2002). In particular, the ERK1/2 MAP kinase pathway is involved in the proliferation of a variety of cell types (Yang et al., 2010). p38 MAP kinase activation results in the downstream expressional activation of several osteoclast marker genes, c-Fos, tartrate-resistant acid phosphatase (TRAP, encoded by Acp5) and cathepsin K (CtsK) during differentiation (Li et al., 2002; Mansky et al., 2002).

For the maturation of osteoclasts and for their bone-resorbing function, osteoclast precursors become from mononuclear cells to matured, multinucleated cells through a cell-cell fusion process (Blare, 1998; Vignery, 2000). Molecules participating in the fusion can be divided into two types: RANKL-independent molecules and RANKL-dependent molecules (Xing et al., 2012; Zhang et al., 2014). Whereas CD44 and TREM2 are not regulated by RANKL (de Vries et al., 2005; Kukita et al., 2004; Xing et al., 2012), CD9/CD81, ATP6v0d2, and DC-STAMP have been shown to be regulated by it (Kim et al., 2008; Kukita et al., 2004; Ishii et al., 2006).

Dendritic cell-specific transmembrane protein (DC-STAMP) and the d2 isoform of vacuolar $\left(\mathrm{H}^{+}\right)$ATPase (v-ATPase) Vo domain (Atp6v0d2) are known to be key regulators for osteoclast maturation, and the deficiency of DC-STAMP or Atp6v0d2 leads to osteopetrosis in mice (Kim et al., 2008; Kukita et al.,

elSSN: 0219-1032

(c) The Korean Society for Molecular and Cellular Biology. All rights reserved.

(C) This is an open-access article distributed under the terms of the Creative Commons Attribution-NonCommercial-ShareAlike 3.0 Unported License. To view a copy of this license, visit http://creativecommons.org/licenses/by-nc-sa/3.0/. 
2004; Lee et al., 2006). Dendritic cell-specific transmembrane protein (DC-STAMP), discovered in human dendritic cells (DCs), is preferentially expressed in osteoclasts rather than DCs and macrophages (Hartgers et al., 2000; Kukita et al., 2004; Rho et al., 2002; Staege et al., 2001; Yagi et al., 2005). Multiple isoforms of some subunits in V-ATPase, including B, C, $E$, and $G$ of $V 1$ and $a$ and $d$ of V0, have been identified in various tissues and cell types (Lee et al., 2006; Nishi et al., 2003; Wu et al., 2009). A 38-kDa protein, Atp6v0d2 (d2), has been found to be expressed in various mammalian tissues and is the most abundant in osteoclasts (Nishi et al., 2003; Wu et al., 2009). This subunit may also contribute to the efficient coupling of ATP hydrolysis of V1 and proton translocation of V0 (Nishi et al., 2003; Wu et al., 2009).

Obatoclax, a synthetic derivative of bacterial prodiginines, binds to all anti-apoptotic Bcl-2 family members and has a role as a pan-Bcl-2 inhibitor (Kim et al., 2014; Konopleva et al., 2008). It has already been used in early clinical trials and has been widely tested in solid malignancies and lymphoma (Campa's et al., 2006; Kim et al., 2014; Konopleva et al., 2008; Trudel et al., 2007). We have recently, for the first time, demonstrated that obatoclax stimulates apoptosis through the suppression of Bcl2A1 by insulin and inhibits the proliferation of osteoclast precursors (Lee and Lee, 2014). Thus, the effects of obatoclax on the proliferation and differentiation of osteoclast precursors have not been well-defined yet.

Based on this earlier report, our present study aimed at investigating the potential role and regulatory mechanisms of obatoclax on proliferation and differentiation of osteoclast precursors. Ultimately, our data revealed that obatoclax suppresses the proliferation of osteoclast precursors by increasing apoptosis, while decreasing the cell fusion through the inhibition of the activation of ERK1/2 MAP kinase by RANKL, showing the potential of obatoclax for the treatment of several bone-related diseases in the future.

\section{MATERIALS AND METHODS}

\section{Isolation of osteoclast precursors}

Isolation of osteoclast precursors and the in vitro osteoclastogenesis experiment were performed as described previously (Choi et al., 2013). In brief, bone marrow cells were flushed out from the femurs of 4-6-week-old C57BL/6 mice with a sterile 21-gauge syringe and incubated in alpha-MEM media containing 10\% FBS and $10 \mathrm{ng} / \mathrm{ml} \mathrm{M-CSF} \mathrm{(R \& D} \mathrm{Systems).}$ After $24 \mathrm{~h}$, non-adherent cells were harvested and cultured in the presence of M-CSF ( $20 \mathrm{ng} / \mathrm{ml})$ for 3 days. After washing out the non-adherent cells, adherent cells were used as osteoclast precursors.

\section{Cell viability (MTT) assay}

Cells were seeded in 96-well plates with $2 \times 104$ cells/well in complete culture medium. After $24 \mathrm{~h}$, the cells were treated with indicated doses of obatoclax (Selleck Chemicals) in the presence or absence of RANKL (Peprotech) for $24 \mathrm{~h}$. Cell viability was measured using the MTT (3-[4,5-dimethyl-2-thiazolyl]-2,5diphenyl-2H-tetrazolium bromide) assay kit (Sigma) and the absorbance was on a microplate reader (Bio-Rad) at $570 \mathrm{~nm}$ (Choi et al., 2013).

\section{BrdU incorporation assay}

The BrdU incorporation assay was performed as described previously (Lee and Lee, 2014). In brief, after $24 \mathrm{~h}$ of seeding with $2 \times 10^{4}$ cells/ well in 96-well plates, the cells were treated with obatoclax or PD98059 (Sigma, USA). Cell proliferation was assayed using the BrdU incorporation assay kit (Cell Signaling Technology) according to the manufacturer's instructions. BrdU was added $12 \mathrm{~h}$ before culture termination. At the end of culture, cells were fixed with fixing solution for $30 \mathrm{~min}$ at RT, rinsed twice with PBS, incubated with monoclonal anti-BrdU antibody for $1 \mathrm{~h}$, followed by anti-mouse secondary antibody for $30 \mathrm{~min}$. After the final wash, the substrate was added to the wells and then the stop solution was administered. The proportion of total cells incorporating BrdU into the nucleus was determined by reading the absorbance on a microplate reader (Bio-Rad) at $450 \mathrm{~nm}$ to calculate cell viability.

\section{Apoptosis assay}

Apoptosis was measured using the Annexin V Apoptosis Kit (BD Biosciences) in accordance with the manufacturer's instructions. Osteoclast precursors were seeded with $2 \times 10^{4}$ cells/ well in 96-well plates and then treated with obatoclax or PD98059. The cells were then washed with cold PBSs, resuspended in $1 \times$ Annexin $\mathrm{V}$ binding buffer (BD Biosciences), and labeled with $10 \mu$ l Annexin V-APC (BD Biosciences) for $15 \mathrm{~min}$ in the dark. After incubation, $1 \times$ binding buffer was added. The analyses were performed using a FACScan flow cytometer (Becton Dickinson) (Lee and Lee, 2014).

\section{Western blot analyses}

Western blotting was performed as described previously (Choi et al., 2013). In brief, osteoclast precursors stimulated with RANKL or obatoclax were lysed in lysis a buffer and the supernatants were prepared by centrifugation, electrophoresed on a $10 \%$ SDS-polyacrylamide gel and blotted onto a polyvinylidene difluoride membrane. Immunoblotting was performed with antibodies (Abs) specific to phosphorylated ERK1/2 (p-ERK1/2), ERK1/2, phosphorylated JNK1/2 (p-JNK1/2), JNK1/2, phosphorylated p38 (p-p38), p38 MAP kinase (Cell Signaling Technology, USA), DC-STAMP, Atp6v0d2, and beta-actin (Santa Cruz Biotechnology, USA), followed by HRP-conjugated secondary antibodies and enhanced using an ECL detection kit (Amersham Biosciences).

\section{In vitro osteoclastogenesis}

For the osteoclast formation assay, the cells isolated from the femur of mice were further cultured in the presence of 200 $\mathrm{ng} / \mathrm{ml}$ of RANKL and $30 \mathrm{ng} / \mathrm{ml}$ of M-CSF. After 3 days, the cells were fixed, stained for tartrate-resistant acid phosphatase (TRAP) using the TRAP staining kit (Sigma). TRAP-positive multinucleated cells (TRAP ${ }^{+}$MNCs) larger than $100 \mu \mathrm{m}$ in diameter containing more than 20 nuclei and $\mathrm{TRAP}^{+}$mononuclear cells were counted, and the number was presented as relative percentage $(\%)$.

\section{Real-time PCR}

Total RNAs isolated from cells were reverse transcribed using SuperscriptIII reverse transcriptase (Invitrogen) according to the manufacturer's protocol. Real-time PCRs were performed with the Brilliant UltraFast SYBR Green QPCR Master Mix (Agilent Technologies) and specific primers for target genes and HPRT (for endogenous control) from QIAGEN in triplicate on an MX3000 instrument (Agilent Technologies). HPRT was used for the normalization of all quantitation (Kim and Lee, 2014).

\section{Statistical analysis}

The results are presented by mean \pm SD of triplicates from at least three independent experiments. Statistical analyses were 
A

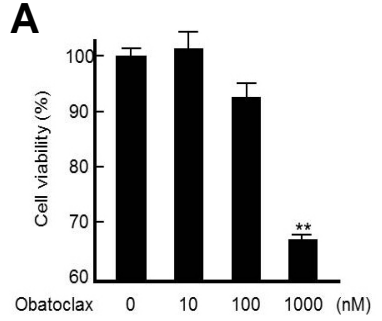

$B$

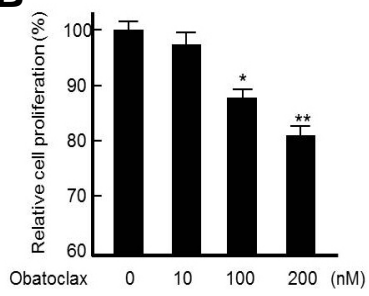

C

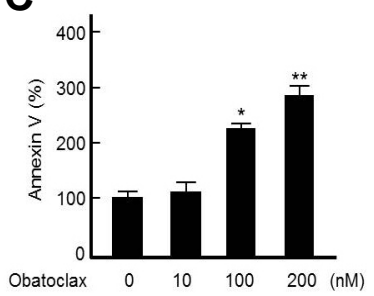

Fig. 1. Effect of obatoclax on cell viability. (A) Osteoclast precursors were incubated with the indicated concentrations of obatoclax for $24 \mathrm{~h}$. The cytotoxicity of obatoclax was determined using the MTT assay kit. (B) The indicated concentrations of obatoclax were treated into the osteoclast precursors for $48 \mathrm{~h}$. Then, cell proliferation was measured using

the BrdU incorporation assay kit. (C) The increase of apoptosis by the indicated concentrations of obatoclax after $48 \mathrm{~h}$ of treatment on osteoclast precursors. Apoptosis was measured using the Annexin V Apoptosis Kit as described in "Materials and Methods". Results are representative of at least three independent experiments. ${ }^{*} p<0.05$ and ${ }^{\star \star} p<0.005$ vs. non-treated cells.

determined using Student's $t$-test. $P<0.05$ was considered significant.

\section{RESULTS}

Obatoclax inhibits the proliferation of osteoclast precursors by inducing apoptosis

Prior to investigating the role of obatoclax on the proliferation and differentiation of osteoclast precursors, we first examined the cytotoxicity of obatoclax. Whereas low doses (10 nM and $100 \mathrm{nM}$ ) of obatoclax did not show a cytotoxic effect, the higher dose (1000 nM) of obatoclax completely decreased cell viability (Fig. 1A). The BrdU incorporation assay showed that the low dose of obatoclax has impressive inhibitory effects on their proliferation (Fig. 1B). On the contrary, obatoclax significantly increased the number of apoptotic cells in a dose-dependent manner (Fig. 1C). These results demonstrate that obatoclax inhibits cell proliferation by inducing the apoptosis of osteoclast precursors.

\section{ERK MAP kinase activation is involved in the proliferation} and apoptosis of osteoclast precursors

To gain insight into how obatoclax regulates proliferation of osteoclast precursors, we examined whether obatoclax regulates the activation of MAP kinases by RANKL. As expected, RANKL effectively activated ERK1/2, JNK1/2, and p38 MAP kinases (Fig. 2A). Interestingly, pretreatment with obatoclax dramatically inhibited the activation of ERK $1 / 2$ by RANKL. However, the activation of JNK1/2 and p38 MAP kinases was not affected (Fig. 2A).

To verify whether the inhibition of ERK1/2 MAP kinase by obatoclax is involved in the proliferation of the cells, an inhibitor of ERK, PD98059, was administered. Whereas RANKL alone failed to regulate cell proliferation and apoptosis, pretreatment with PD98059 effectively suppressed cell proliferation and increased apoptosis in the presence of RANKL, just as obatoclax did (Figs. 2B and $2 \mathrm{C}$ ). These results suggest that obatoclax regulates the proliferation and apoptosis of osteoclast precursors through the inhibition of ERK1/2 MAP kinase.

Obatoclax down-regulates the fusion of osteoclast precursors by blocking the ERK1/2 signaling pathway

It is known that the activation of ERK1/2 MAP kinase is important for the osteoclast differentiation induced by RANKL (Boyle et al., 2003). Prior to determining whether the inhibition of ERK1/2 MAP kinase by obatoclax affects osteoclast differentiation, we first examined the effect of PD98059 on the cells. Stimulation with RANKL markedly induced osteoclast differentiation (Figs. 3A and 3D). However, pretreatment with PD98059
$\boldsymbol{A}$
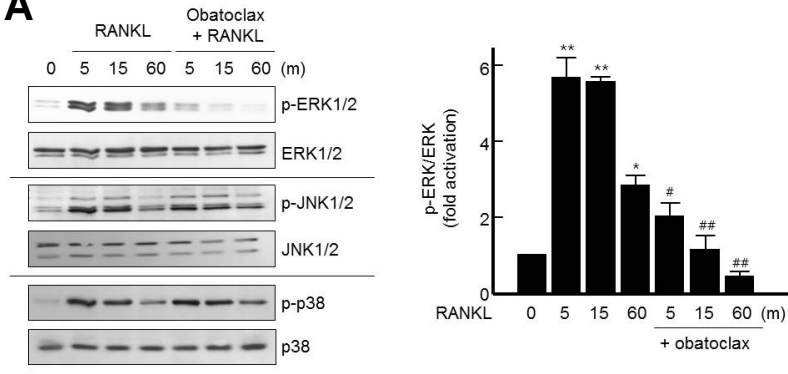

B

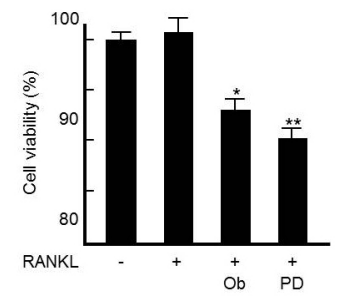

C

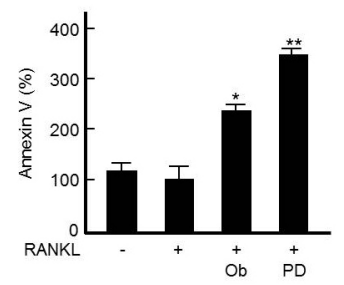

Fig. 2. ERK MAP kinase activation is involved in the proliferation and apoptosis of osteoclast precursors. (A) Osteoclast precursors were pretreated with or without $100 \mathrm{nM}$ of obatoclax before $2 \mathrm{~h}$ of RANKL treatment for the indicated times. Total proteins were isolated and then subjected to Western blot analyses using MAP kinases-specific antibodies (left). Protein bands were quantified by densitometry, and the level of phosphorylated ERK1/2 MAP kinase was normalized to that of ERK1/2 (right). ( $B$ and $C$ ) Osteoclast precursors were pretreated with obatoclax $(100 \mathrm{nM}, \mathrm{Ob})$ before $2 \mathrm{~h}$ or PD98059 (10 $\mu \mathrm{M}, \mathrm{PD})$ before $30 \mathrm{~min}$ of RANKL treatment for 48 h. Then, cell proliferation $(B)$ and apoptosis $(C)$ were measured as described in Materials and Methods. Results are representative of at least three independent experiments. ${ }^{*} p<0.05$ and ${ }^{* *} p<0.005$ vs. non-treated cells.

completely suppressed the number of fused, multinucleated cells larger than $100 \mu \mathrm{m}$ in diameter containing more than 20 nuclei, whereas the number of TRAP-positive mononuclear cells was increased (Figs. 3B and $3 \mathrm{C}$ ). Obatoclax showed a similar effect to the ERK inhibitor (Figs. 3E and 3F). However, obatoclax alone failed to regulate osteoclast differentiation (Fig. 3D). These results indicate that obatoclax suppresses the fusion of osteoclast precursors into multinucleated cells through the inhibition of ERK1/2 MAP kinase. 
A

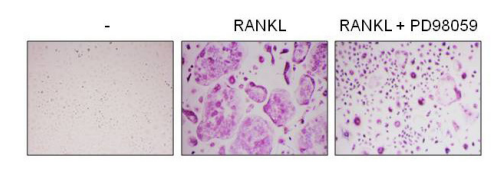

D

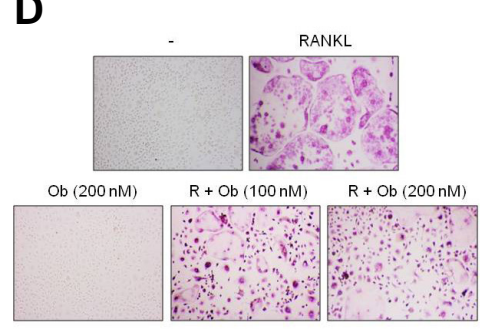

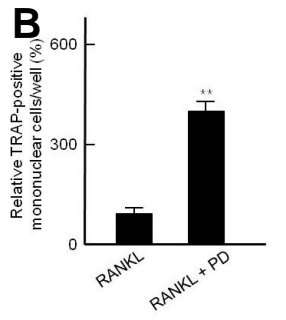

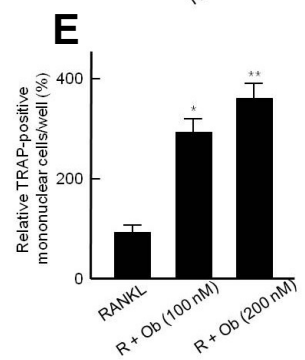

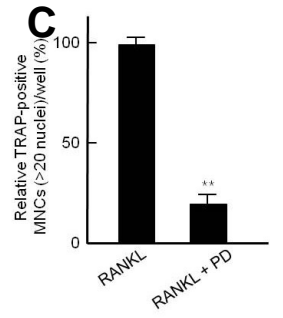

$\boldsymbol{F}$

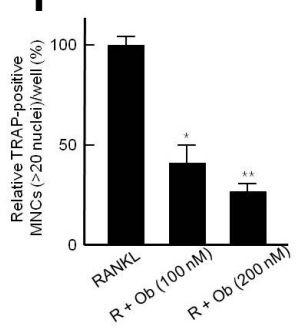

Fig. 3. Obatoclax down-regulates the fusion of osteoclast precursors through the ERK1/2 MAP kinase. (A-C) Osteoclast precursors were pretreated with $10 \mu \mathrm{M}$ of PD98059 for $30 \mathrm{~min}$ and then RANKL for 3 days. TRAP staining was performed (original magnification, $\times 100)(A)$ and TRAP $^{+}$mononuclear cells (B) or $\mathrm{TRAP}^{+}$MNCs larger than $100 \mu \mathrm{m}$ in diameter containing more than 20 nuclei $(C)$ were counted and presented as relative percentage (\%). ${ }^{*} p<0.05$ and ${ }^{* *} p<0.005$ vs. RANKLtreated cells. (D-F) As in (A-C), except that $100 \mathrm{nM}$ or $200 \mathrm{nM}$ of obatoclax $(\mathrm{Ob})$ for $2 \mathrm{~h}$ was pretreated instead of PD98059.
$\boldsymbol{A}$
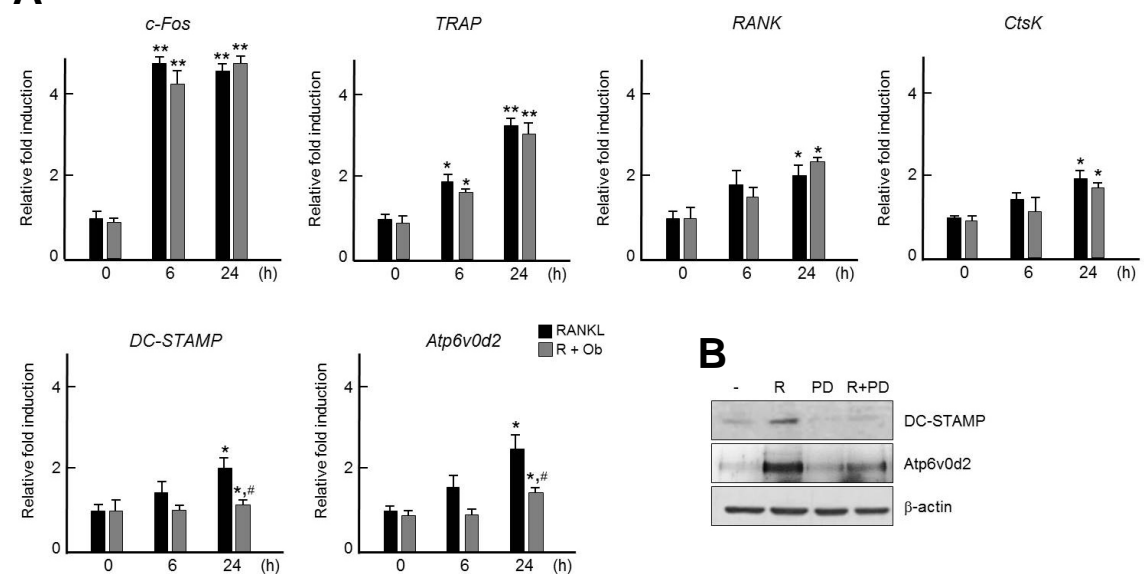

Fig. 4. Obatoclax decreases the expression of DC-STAMP and Atp6v0d2. (A) Isolated osteoclast precursors were pretreated with $100 \mathrm{nM}$ of obatoclax $(\mathrm{Ob})$ for $2 \mathrm{~h}$ and then RANKL $(\mathrm{R})$ for the indicated times. Total RNA was isolated from the cells and then subjected to real-time PCR as described in "Materials and Methods". All quantitation was normalized to an HPRT and the fold increase in the stimulated cells was compared with untreated cells as shown. Results are representative of at least three independent experiments. ${ }^{*} p<0.05$ and ${ }^{* *} p<0.005$ vs. non-treated cells. $\# p<0.05$ vs. RANKLtreated cells for $24 \mathrm{~h}$. (B) Osteoclast precursors were pretreated with $10 \mu \mathrm{M}$ of PD98059 (PD) for $30 \mathrm{~min}$ then treated with RANKL (R) for 3 days. $30 \mu \mathrm{g}$ of total cell lysates was analyzed by Western blot analysis.
Obatoclax suppresses the expression of DC-STAMP and Atp6v0d2 by RANKL

The result that obatoclax down-regulated the fusion of osteoclast precursors led us to hypothesize that obatoclax may control the expression of DC-STAMP and Atp6v0d2, which are both known to regulate the cell-cell fusion (Kukita et al., 2004; Lee et al., 2006), of osteoclast precursors. To verify that, we examined the expression of osteoclast marker genes including DC-STAMP and Atp6v0d2 was affected by obatoclax treatment. While RANKL effectively induced the expression of the osteoclast marker genes, c-Fos, TRAP, RANK, and CtsK, pretreatment with obatoclax did not affect their expression (Fig. 4A). However, the expression of DC-STAMP and Atp6v0d2 induced by RANKL stimulation was abolished by obatoclax pretreatment (Fig. 4A).

To determine whether this result was caused by the inhibition of ERK1/2 MAP kinase, cells were pretreated with PD98059. Western blot analyses showed that pretreatment with PD98059 completely inhibited the expression of DC-STAMP and Atp6v0d2 induced by RANKL (Fig. 4B), which strongly supports that obatoclax regulates the fusion of osteoclast precursors through the ERK 1/2 MAP kinase pathways, a finding consistent with previous data (Fig. 3). Taken together, these data suggest that obatoclax regulates the proliferation and fusion of osteoclast precursors negatively through the inhibition of the ERK1/2 MAP kinase pathway by RANKL (Fig. 5).

\section{DISCUSSION}

The Bcl2/Bax ratio determines cell fate: proliferation or apoptosis. Whereas an enhancement of $\mathrm{Bcl} 2$, an anti-apoptotic protein, promotes cell proliferation, Bax, a pro-apoptotic protein, activates caspase 3 and induces apoptosis (Hockenbery et al., 1993; Yamashita et al., 2008). Bcl2 ${ }^{/-}$mice showed increased bone mass due to the induced apoptosis of osteoclasts; however, proliferation and differentiation of osteoblasts are not affected (Yamashita et al., 2008). This suggests that Bcl2 plays a critical anti-apoptotic role in osteoclasts rather than osteoblasts. Obatoclax, a pan-Bcl2 inhibitor, has been applied in various solid malignancies and lymphoma, and exerted antitumor activities (Hwang et al., 2010; Paik et al., 2010). Despite the importance of Bcl2 in osteoclasts, the effects of obatoclax on the 


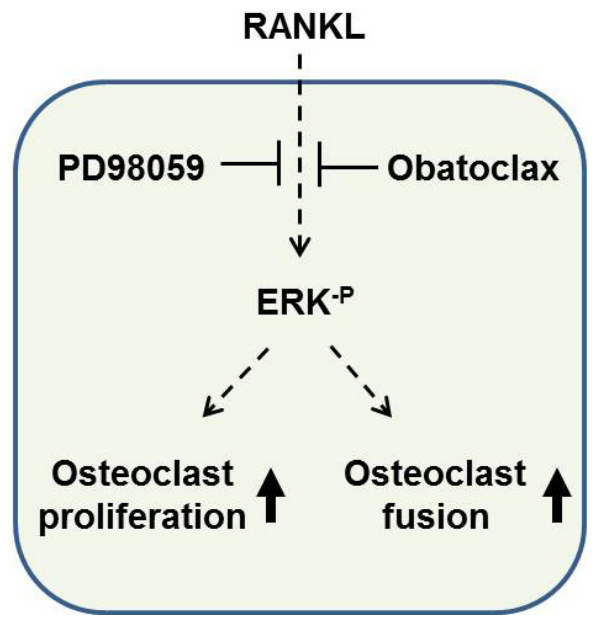

Fig. 5. The regulatory mechanisms model of the proliferation and fusion of osteoclast precursors by obatoclax. Obatoclax inhibits the ERK1/2 MAP kinase activation by RANKL, thus suppressing the proliferation and fusion of osteoclast precursors.

proliferation and differentiation of osteoclast precursors have not been well-defined. We have recently shown that obatoclax suppresses their proliferation by insulin through the inhibition of apoptosis (Lee and Lee, 2014). Based on this previous study, the present study aimed to assess the potential of obatoclax on the proliferation and differentiation of osteoclast precursors. Here, we describe the anti-proliferative effects of obatoclax on osteoclast precursors and its negative role in their fusion.

Stimulation with low doses of obatoclax significantly suppressed the cell proliferation in a dose-dependent manner, while markedly increasing the apoptosis of osteoclast precursors. These functions were found to be through ERK MAP kinase signaling since its stimulation was sufficient to block the activation of ERK MAP kinase by RANKL and these obvious effects were also observed when PD98059, an ERK inhibitor, was administered in the cells.

The ERK MAP kinase signaling pathway seems to be related to two distinct mechanisms in osteoclast precursors: on one hand, by stimulating proliferation through the down-regulation of apoptosis, and on the other hand, by up-regulating the cell fusion, thus contributing to osteoclastogenesis. Pretreatment with either obatoclax or PD98059 dramatically reduced the cellcell fusion by RANKL stimulation via blocking of the ERK signaling pathway. However, TRAP-positive mononuclear cells were relatively increased compared to when RANKL alone was treated since RANKL markedly increased the number of multinucleated cells (TRAP ${ }^{+}$MNCs) larger than $100 \mu \mathrm{m}$ in diameter containing more than 20 nuclei although obatoclax and PD98059 inhibited the proliferation of osteoclast precursors.

Interestingly, the activation of JNK1/2 and p38 MAP kinase, necessary for osteoclast differentiation, by RANKL was not affected by obatoclax. These results are consistent with the finding that obatoclax failed to regulate the expression of osteoclast marker genes, including c-Fos, TRAP, RANK and CtsK. Instead, the expression of DC-STAMP and Atp6v0d2 by RANKL was significantly reduced by obatoclax, which was through the ERK pathway since PD98059 completely abrogated the expression of these two proteins. These results imply that obatoclax may work during the late stage of osteoclastogenesis undergoing cell-cell fusion, rather than the earlier stage of osteoclast differentiation.

Still, there are the subjects of further research: 1) whether obatoclax results in the arrest of the cell cycle in osteoclast precursors, and 2) whether or how Bcl2 and ERK signaling is connected in the regulation of the proliferation and differentiation of the cells. In the previous study, cyclinD1, a cell cycle regulator, was affected by insulin stimulation in osteoclast precursors, and thus enhanced cell proliferation (Lee and Lee, 2014). This shows that cyclinD1 may be a target of obatoclax through the ERK signaling pathway. It needs to be further studied.

Continued investigation into the signaling and molecular mechanisms of obatoclax on osteoclast precursors could contribute to the understanding of bone metabolism and give hints toward novel targets for treatment.

\section{ACKNOWLEDGMENTS}

This work was supported by Basic Science Research Program through the National Research Foundation of Korea funded by the Ministry of Education, Science and Technology (NRF-20100025761) and the Soonchunhyang University Research Fund.

\section{REFERENCES}

Anderson, D.M., Maraskovsky, E., Billingsley, W.L., Dougall, W.C., Tometsko, M.E., Roux, E.R., Teepe, M.C., DuBose, R.F., Cosman, D., and Galibert, L. (1997). A homologue of the TNF' receptor and its ligand enhance T-cell growth and dendritic-cell function. Nature 390, 175-179.

Blair, H.C. (1998). How the osteoclast degrades bone. Bioessays 20, 837-848.

Boyle, W.J., Simonet, W.S., and Lacey, D.L. (2003). Osteoclast differentiation and activation. Nature 423, 337-342.

Campa's, C., Cosialls, A.M., Barragán, M., Iglesias-Serret, D., Santidrián, A.F., Coll-Mulet, L., de Frias, M., Domingo, A., Pons, G., and Gil, J. (2006). Bcl-2 inhibitors induce apoptosis in chronic lymphocytic leukemia cells. Exp. Hematol. 34, 1663-1669.

Choi, J., Choi, S.Y., Lee, S.Y., Lee, J.Y., Kim, H.S., Lee, S.Y., and Lee, N.K. (2013). Caffeine enhances osteoclast differentiation and maturation through p38 MAP kinase/Mitf and DCSTAMP/CtsK and TRAP pathway. Cell. Signal. 25, 1222-1227.

David, J.P., Sabapathy, K., Hoffmann, O., Idarraga, M.H., and Wagner, E.F. (2002). JNK1 modulates osteoclastogenesis through both C-Jun phosphorylation-dependent and independent mechanisms. J. Cell Sci. 115, 4317-4325.

de Vries, T.J., Schoenmaker, T., Beertsen, W., van der Neut, R., and Everts, V. (2005). Effect of CD44 deficiency on in vitro and in vivo osteoclast formation. J. Cell. Biochem. 94, 954-966.

Harada, S., and Rodan, G.A. (2003). Control of osteoblast function and regulation of bone mass. Nature 423, 349-355.

Hartgers, F.C., Vissers, J.L., Looman, M.W., van Zoelen, C., Huffine, C., Figdor, C.G., and Adema, G.J. (2000). DC-STAMP, a novel multimembrane-spanning molecule preferentially expressed by dendritic cells. Eur. J. Immunol. 30, 3585-3590.

Hockenbery, D.M., Oltvai, Z.N., Yin, X.M., Milliman, C.L., and Korsmeyer, S.J. (1993). Bcl-2 functions in an antioxidant pathway to prevent apoptosis. Cell 75, 241-251.

Hwang, J.J., Kuruvilla, J., Mendelson, D., Pishvaian, M.J., Deeken, J.F., Siu, L.L., Berger, M.S., Viallet, J., and Marshall, J.L. (2010). Phase I dose finding studies of obatoclax (GX15-070), a small molecule pan-BCL-2 family antagonist, in patients with advanced solid tumors or lymphoma. Clin. Cancer Res. 16, 40384045.

Ishii, M., Iwai, K., Koike, M., Ohshima, S., Kudo-Tanaka, E., Ishii, T., Mima, T., Katada, Y., Miyatake, K., Uchiyama, Y., and Saeki, Y. (2006). RANKL-induced expression of tetraspanin CD9 in lipid raft membrane microdomain is essential for cell fusion during osteoclastogenesis. J. Bone Miner. Res. 21, 965-976.

Kim, H.S., and Lee, N.K. (2014). Gene expression profiling in osteoclast precursors by insulin using microarray analysis. Mol. Cells 
30, 827-832

Kim, K., Lee, S.H., Kim, J., Choi, Y., and Kim, N. (2008). NFATc1 induces osteoclast fusion via upregulation of Atp6v0d2 and the dendritic cell-specific transmembrane protein (DCSTAMP). Mol. Endocrinol. 22, 176-185.

Kim, P.S., Jochems, C., Grenga, I., Donahue, R.N., Tsang, K.Y., Gulley, J.L., Schlom, J., and Farsaci, B. (2014). Pan-Bcl-2 Inhibitor, GX15-070 (Obatoclax), decreases human T regulatory lymphocytes while preserving effector $T$ lymphocytes: a rationale for its use in combination immunotherapy, J. Immunol. 192, 26222633.

Konopleva, M., Watt, J., Contractor, R., Tsao, T., Harris, D., Estrov, Z., Bornmann, W., Kantarjian, H., Viallet, J., Samudio, I., et al (2008). Mechanisms of antileukemic activity of the novel Bcl-2 homology domain-3 mimetic GX15-070 (obatoclax). Cancer Res. 68, 3413-3420.

Kukita, T., Wada, N., Kukita, A., Kakimoto, T., Sandra, F., Toh, K., Nagata, K., lijima, T., Horiuchi, M., Matsusaki, H., et al. (2004). RANKL-induced DC-STAMP is essential for osteoclastogenesis. J. Exp. Med. 200, 941-946.

Lee, J.Y., and Lee, N.K. (2014). Up-regulation of cyclinD1 and Bcl2A1 by insulin is involved in osteoclast proliferation. Life Sci. 114, 57-61.

Lee, S.H., Rho, J., Jeong, D., Sul, J.Y., Kim, T., Kim, N., Kang, J.S., Miyamoto, T., Suda, T., Lee, S.K., et al. (2006). v-ATPase VO subunit d2-deficient mice exhibit impaired steoclast fusion and increased bone formation. Nat. Med. 12, 1403-1409.

Li, X., Udagawa, N., Itoh, K., Suda, K., Murase, Y., and Nishihara, T. (2002). p38 MAPK-mediated signals are required for inducing osteoclast differentiation but not for osteoclast function. Endocrinology 143, 3105-3113.

Mansky, K.C., Sankar, U., Han, J., and Ostrowski, M.C. (2002). Microphthalmia transcription factor is a target of the p38 MAPK pathway in response to receptor activator of NF- B ligand signaling. J. Biol. Chem. 277, 11077-11083.

Nishi, T., Kawasaki-Nishi, S., and Forgac, M. (2003). Expression and function of the mouse V-ATPase d subunit isoforms. J. Biol. Chem. 278, 46396-46402.

Paik, P.K., Rudin, C.M., Brown, A., Rizvi, N.A., Takebe, N., Travis, W., James, L., Ginsberg, M.S., Juergens, R., Markus, S., et al. (2010). A phase I study of obatoclax mesylate, a Bcl-2 antagonist, plus topotecan in solid tumor malignancies. Cancer Chemother. Pharmacol. 66, 1079-1085.

Rho, J., Altmann, C.R., Socci, N.D., Merkov, L., Kim, N., So, H.,
Lee, O., Takami, M., Brivanlou, A.H., and Choi, Y. (2002). Gene expression profiling of osteoclast differentiation by combined suppression subtractive hybridization (SSH) and cDNA microarray analysis. DNA Cell. Biol. 21, 541-549.

Rodan, G.A., and Martin, T.J. (2000) Therapeutic approaches to bone diseases. Science 289, 1508-1514.

Staege, H., Brauchlin, A., Schoedon, G., and Schaffner, A. (2001) Two novel genes FIND and LIND differentially expressed in deactivated and Listeria-infected human macrophages. Immunogenetics 53, 105-113.

Teitelbaum, S.L. (2000). Bone resorption by osteoclasts. Science 289, 1504-1508.

Trudel, S., Li, Z.H., Rauw, J., Tiedemann, R.E., Wen, X.Y., and Stewart, A.K. (2007). Preclinical studies of the pan-Bcl inhibitor obatoclax (GX015-070) in multiple myeloma. Blood 109, 54305438.

Vignery, A. (2000). Osteoclasts and giant cells: macrophagemacrophage fusion mechanism. Int. J. Exp. Pathol. 81, 291-304

Wei, S., Wang, M.W., Teitelbaum, S.L., and Ross, F.P. (2002). Interleukin-4 reversibly inhibits osteoclastogenesis via inhibition of NF-kappa B and mitogen-activated protein kinase signaling. J. Biol. Chem. 277, 6622-6630.

Wong, B.R., Josien, R., Lee, S.Y., Sauter, B., Li, H.L., Steinman, R.M., and Choi, Y. (1997). TRANCE (tumor necrosis factor [TNF]-related activation-induced cytokine), a new TNF family member predominantly expressed in T cells, is a dendritic cellspecific survival factor. J. Exp. Med. 186, 2075-2080.

Wu, H., Xu, G., and Li, Y.P. (2009). Atp6v0d2 is an essential component of the osteoclast-specific proton pump that mediates extracellular acidification in bone resorption. J. Bone Miner. Res. 24, 871-885.

Xing, L., Xiu, Y., and Boyce, B.F. (2012). Osteoclast fusion and regulation by RANKL-dependent and independent factors. World J. Orthop. 3, 212-222.

Yamashita, J., Datta, N.S., Chun, Y.H., Yang, D.Y., Carey, A.A Kreider, J.M., Goldstein, S.A., and McCauley, L.K. (2008). Role of Bcl2 in osteoclastogenesis and PTH anabolic actions in bone. J. Bone Miner. Res. 23, 621-632.

Yang, J., Zhang, X., Wang, W., and Liu, J. (2010). Insulin stimulates osteoblast proliferation and differentiation through ERK and PI3K in MG-63 cells. Cell Biochem. Funct. 28, 334-341.

Zhang, C., Dou, C.E., Xu, J., and Dong, S. (2014). DC-STAMP, the key fusion-mediating molecule in osteoclastogenesis. J. Cell. Physiol. 229, 1330-1335. 